\title{
Serum uric acid: a biochemical prognostic indicator of pregnancy outcomes among pre-eclampsia patients at the federal medical centre, Yenagoa
}

\author{
Lukman Obagah $^{1 *}$, Benedicta E. Kasia ${ }^{2}$, Israel Jeremiah ${ }^{1}$, Dennis O. Allagoa1, \\ Egbaname E. O. S. Aigere ${ }^{1}$, Ebikabowei L. Kotingo ${ }^{1}$, Chibuzor P. Oriji ${ }^{1}$
}

\author{
${ }^{1}$ Department of Obstetrics and Gynaecology, Federal Medical Centre, Yenagoa, Bayelsa State, Nigeria \\ ${ }^{2}$ Department of Chemical Pathology, Niger Delta University Teaching Hospital, Okolobiri, Bayelsa State, Nigeria
}

Received: 27 June 2020

Revised: 04 August 2020

Accepted: 15 September 2020

\author{
*Correspondence: \\ Dr. Lukman Obagah, \\ E-mail: obagahlukeman@gmail.com
}

Copyright: (C) the author(s), publisher and licensee Medip Academy. This is an open-access article distributed under the terms of the Creative Commons Attribution Non-Commercial License, which permits unrestricted non-commercial use, distribution, and reproduction in any medium, provided the original work is properly cited.

\begin{abstract}
Background: High levels of uric acid concentration over normal pregnant values have been reported previously in established preeclampsia, hence it was suggested to be a useful biochemical prognostic marker of the disease.

Methods: This is a hospital based prospective case control study where 100 patients with preeclampsia were recruited consecutively as they were admitted. 100 non-pre-eclamptics were also recruited as control. The foetomaternal data, adverse perinatal outcomes, maternal serum uric acid levels as well as complications of preeclampsia were recorded into the protocol. Statistical analysis was done using SPSS 22.0. Level of significance was set at $\mathrm{p}<0.05$.

Results: The mean age in the study group was $28 \pm 6.7$ years while in the control group it was $31 \pm 6.5$ years. The difference in age was not statistically significant $(\mathrm{p}=0.53)$. The mean serum uric acid level was significantly higher amongst participants with preeclampsia than in those without preeclampsia $(405.6 \pm 995 \mu \mathrm{mol} / \mathrm{L}$ versus $232.7 \pm 26.3$ $\mu \mathrm{mol} / \mathrm{L}, \mathrm{p}=0.00)$. Uric acid was statistically associated with the occurrence of eclampsia $(\mathrm{p}=0.03)$, Severe hypertension $(0.03)$ and birth asphyxia $(\mathrm{p}=0.01)$.

Conclusions: The mean serum uric acid was higher in preeclampsia than non-preeclampsia patients. High levels of uric acid were found in eclampsia, severe hypertension and birth asphyxia. However, the prognostic accuracy for uric acid was low for most pregnancy outcomes.
\end{abstract}

Keywords: Pre-eclampsia, Eclampsia, Uric acid, Pregnancy outcomes

\section{INTRODUCTION}

Preeclampsia has remained a major challenge to obstetric practice and it is a common complication of pregnancy associated with high maternal and perinatal morbidity and mortality especially in developing countries. ${ }^{1}$ Preeclampsia is a pregnancy associated multiorgan disorder caused by altered trophoblastic invasion and endothelial cell dysfunction. ${ }^{2}$ It accounts for about 12$18 \%$ of pregnancy related deaths. ${ }^{2}$ Preeclampsia is a pregnancy specific disease characterized by hypertension and proteinuria arising de novo after the 20th week of gestation in a previously normotensive and non proteinuric woman. The exact cause of preeclampsia remains unclear. The central pathology to the development of preeclampsia lies in the placenta. ${ }^{4}$ The incidence is higher in conditions with increased placental mass like twin pregnancy, molar pregnancy, diabetic pregnancies and hydrops foetalis. ${ }^{4}$ Genetic and immunological theories have also been implicated. ${ }^{4}$ 
Biochemical indices of preeclampsia include serum calcium, trace elements assay, serum uric acid assay, serum beta human chorionic gonadotropin as well as serum fibronectin. ${ }^{5}$ Serum uric acid is a reliable index of foetal wellbeing when pregnancy is complicated by preeclampsia. With hyperuricaemia, prognosis for the foetus is poor irrespective of level of blood pressure. ${ }^{6}$ It identifies women with increased risk of adverse maternal and particularly foetal outcome. ${ }^{6}$

Uric acid is a product of purine metabolism generated during the breakdown of nucleic acids (DNA and RNA) and adenosine triphosphate. ${ }^{7}$ Its main metabolism is by the kidneys. In normal pregnancy, serum uric acid concentration initially falls by $25-30 \%$ due to elevation in renal clearance secondary to increased glomerular filtration rate or reduced proximal tubular reabsorption due to change in its production rate. ${ }^{8}$ Later in pregnancy, the serum uric acid level increases possibly due to rise in foetal production, decreased binding to albumin, and a decline in uric acid clearance until towards the end of pregnancy when they approach the non-pregnant value. ${ }^{8}$ However, uric acid concentration is increased over normal pregnant values in established preeclampsia and there are several potential origins for raised uric acid concentration in preeclampsia. It is usually secondary to altered renal function, increased tissue breakdown, increased oxidative stress and increased activity of xanthine oxidase. 8 The first report of increased maternal serum uric acid concentration in preeclampsia/eclampsia was published in 1917. ${ }^{9}$ Subsequently some studies reported positive association between elevated uric acid levels and the severity of preeclampsia. Maternal serum uric acid was reported to be useful in the prognostication of the disease and may reflect the severity of the condition. ${ }^{10,11}$ However, others concluded that uric acid levels, although significantly elevated in women with preeclampsia compared with those of normotensive pregnant women, but was not a good prognostic indicator of severity of maternal and foetal complications. ${ }^{12}$

This study revealed serum uric acid as a prognostic indicator among preeclampsia patients and nonpreeclampsia patients and also determined pregnancy outcomes associated with them.

The ability to detect the mild form of preeclampsia would allow closer surveillance and early intervention to improve outcome. ${ }^{13}$ The resultant effect of this study will help reduce the perinatal and maternal morbidity and mortality associated with preeclampsia.

\section{METHODS}

The study was conducted in the Obstetrics and Gynaecology Department of the Federal Medical Centre, Yenagoa. It was a prospective case control study by systematic sampling selection. The first group of the study population comprised 100 consecutive preeclamptic patients admitted for management into our antenatal ward and labour ward. The second group (control) of the study population comprised 100 consecutive non-preeclamptic patients admitted for management into the antenatal ward and labour ward. The values of their serum uric acid were evaluated on admission. Patients were followed up to delivery and their pregnancy outcome evaluated. The study was carried out from the 1st of April 2018 to the 30th of September 2018.

\section{Ethical approval}

Written and informed consent was obtained from every participant in this study. The hospital research and ethics committee examined and approved this research work. Inclusion criteria constituted all preeclampsia patients admitted into the antenatal ward and labour ward of Federal Medical Centre, Yenagoa, who consented to be part of the study within the study period and the controls were normal healthy pregnant women whose serum uric acid levels were assessed within the same period. Exclusion criteria included patients with chronic hypertension, chronic hypertension with superimposed preeclampsia, pregnancy induced hypertension, renal disease, diabetes mellitus, heart failure and ischemic heart disease. Women who refuse to give consent were also excluded from the study.

\section{Sample size}

The sample size for the study was calculated using the formula: ${ }^{14}$

$\mathrm{n}=\mathrm{z}^{2} \mathrm{pq} / \mathrm{d}^{2}$

$\mathrm{n}=$ minimum sample size, $\mathrm{z}=$ standard normal deviation set at $95 \%$ confidence limit $=1.96, p=$ prevalence of preeclampsia in previous study, $q=1-\mathrm{p}$ (complementary probability), $\mathrm{d}=$ margin of error $=5 \%=0.05$

Prevalence of preeclampsia that was used in this study based on a previous study done in Bayelsa state was $5.6 \% .1$ Therefore, giving an attrition of $10 \%$, the minimum sample size was 89. However, this was adjusted to 100 for ease. Thus, 100 patients who met the inclusion criteria were recruited for this study. In our centre, about 400 patients register for antenatal care per month and about 5\%-10\% when followed up to the third trimester are estimated to develop preeclampsia. This study was carried out within 5 months.

Patients were recruited consecutively as they were admitted into the antenatal ward and labour ward with preeclampsia. Thorough history and examination were used in selecting patients based on the inclusion and exclusion criteria. The blood pressure was measured with the use of manual sphygmomanometer. A patient was said to be hypertensive when her blood pressure was persistently equal to or greater than $140 / 90 \mathrm{mmHg}$ measured at least 6 hours apart. 


\section{Specimen collection}

Urine collection was done in the antenatal ward and labour ward. Patients were trained and instructed adequately on how to collect clean catch midstream urine. The urine specimen was taken to the laboratory for protein estimation and in suspicious cases, urine microscopy, culture and sensitivity was done to exclude urinary tract infection. The diagnosis of proteinuria was made when two midstream samples of urine collected at least four hours apart showed one or more plus of albumin using dipstick. ${ }^{15}$ For the diagnosis and classification of preeclampsia, Davey and McGillivray's classification adopted by the international society for the study of hypertension in pregnancy (ISSH) was used. Patients that met the criteria for preeclampsia were recruited into the study. Once the diagnosis was confirmed, blood specimen was collected from the patient for uric acid analysis. Urine specimen and blood specimen were also collected from normal non preeclamptic as controls. About five millilitres of blood was collected. Serum was separated by centrifugation for ten minutes at $3500 \mathrm{rpm}$. The supernatant was transferred by pasteur pipette into a test tube for immediate analysis or stored at $2-8{ }^{\circ} \mathrm{C}$ until time of analysis usually within 24 hours. Analysis of uric acid was done by phosphotungstic acid method and the results were read by spectrophotometer. ${ }^{16}$

\section{Data collection}

Socio-demographic data and clinical characteristics such as age, tribe, marital and booking status was obtained and recorded in the protocol. In addition, the gestational age at delivery, birth weight, 5-minute apgar scores and admission to special care baby unit was noted. Adverse perinatal outcomes like intrauterine growth restriction (IUGR), birth asphyxia and intrauterine foetal death (IUFD) were also noted. Mothers admitted into intensive care unit were followed up and their outcomes recorded. Maternal adverse outcomes such as eclampsia, acute renal failure and HELLP syndrome were also noted. Laboratory results for uric acid and proteinuria were also collected.

\section{Data analysis}

Data entry and statistical analysis was done using statistical package for social science (IBM SPSS for windows version 22.0. SPSS Inc; Chicago, USA).

\section{RESULTS}

\section{Socio-demographic characteristics of participants}

A total of 200 participants were involved in this study which constituted one hundred preeclamptic women (study group) and one hundred non preeclamptic women (control group) with a $100 \%$ response rate. The predominant age group in both the study group and the control group was 25-34 years age group with 52 (52.0\%) and $56(56.0 \%)$ respectively. The mean age in the study group was $28 \pm 6.7$ years, while in the control it was $31 \pm 6.5$ years. The difference in age between the groups was not statistically significant $(p=0.53)$. Majority of the patients were of the Ijaw ethnic group both in the preeclamptic and control groups with $62(62.0 \%)$ and 56 $(56.0 \%)$ respectively. Most of the patients were married in both the preeclamptic and control groups with 84 $(84.0 \%)$ and $92(92.0 \%)$ married respectively. Amongst those that were married, most of the marriages were in the monogamous setting; $78(92.9 \%)$ in the preeclamptic and $89(97.7 \%)$ in the control group. Most of the patients were Christians in both the preeclamptic and control groups with $94(94.0 \%)$ and $96(96.0 \%)$ respectively. The highest level of education was secondary school in the preeclampsia group (58\%), while it was tertiary education for controls (54\%). Majority $56(56 \%)$ and $54(54 \%)$ of the participants in both the preeclamptic and control groups respectively were business women by occupation.

\section{Maternal history and urinalysis dipstick results}

The maternal history and urinalysis dipstick results in both groups revealed that the primipaternity risk was $69 \%$ and $45 \%$ in the preeclamptic and control group respectively. Almost all (98\%) of preeclamptic patients and $100 \%$ of controls were in their third trimester of pregnancy. All the preeclamptic patients had one to three pluses proteinuria as against $3 \%$ in the control group. Thirty-five $(35.0 \%)$ of patients of the study group had a positive family history of pregnancy induced hyper tension (PIH) compared to $7 \%$ in the control group, Amongst those that have positive family history of PIH in the preeclampsia group, $15(42.9 \%)$ said their sisters and they themselves has had PIH in the past, while $5(14.3 \%)$ said their mothers have had PIH previously. In the preeclamptic group and control group, 28 (28.0\%) and 48 $(48.0 \%)$ respectively has had a previous pregnancy. Amongst those that have had a previous pregnancy in the preeclamptic group, $24(85.7 \%)$ carried their pregnancy to term, while, $3(10.7 \%)$ had miscarriages, and $1(3.6 \%)$ had ectopic pregnancy. Amongst those that have had a previous pregnancy in the control group, 35 (72.9\%) carried their pregnancy to term, while $11(22.9 \%)$ had miscarriages, and $2(4.2 \%)$ had ectopic pregnancy.

\section{Maternal symptoms, foetal parameters and maternal adverse pregnancy outcomes}

All the participants with preeclampsia showed symptoms in keeping with the disease as follows; severe nausea and vomiting (42\%), frontal headache (24\%), right upper quadrant epigastric pain $(6 \%)$. None of the control group had such symptoms. Based on maternal pregnancy outcomes in the preeclamptic group, 7\% had HELLP syndrome, $27 \%$ had eclampsia and $10 \%$ developed acute renal failure while none of the controls had such outcomes. Abnormal foetal heart rate was found in $23 \%$ of those with preeclampsia compared to $9 \%$ of the control 
groups. After delivery, the preeclamptic mothers had $40 \%$ of their babies admitted into the special care baby unit, $20(20.0 \%), 10(10.0 \%)$ and $26(26.0 \%)$ of their babies had intrauterine growth retardation, intrauterine foetal death and birth asphyxia respectively. While, in the control group, none of the participant's babies had IUGR, $2(2.0 \%)$ had IUFD and $6(6.0 \%)$ had birth asphyxia.

\section{Mean serum uric acid levels in preeclampsia and control groups}

The mean serum uric acid level $(405.6 \pm 99.5)$ in the preeclampsia group was found to be greater than the mean serum uric acid level in the control group (232.7 \pm 26.3$)$. The observed difference was statistically significant $\mathrm{p}=0.001$, (Table 1$)$.

Table 1: Mean serum uric acid levels in preeclampsia and control groups.

\begin{tabular}{|llllll|}
\hline Group & Mean \pm SD & Mode & Range & t-test & p-value \\
\cline { 1 - 4 } Preeclampsia group & $405.6 \pm 99.5$ & 343 & $421(701-280)$ & & \multirow{2}{*}{0.001} \\
\cline { 1 - 5 } & $232.7 \pm 26.3$ & 213 & $119(290-171)$ & \\
\hline
\end{tabular}

Table 2: Relationship between high maternal serum uric acid levels and maternal/foetal complications in preeclampsia participants.

\begin{tabular}{|llllllll|}
\hline Risk & & & \multicolumn{5}{c|}{ Complications } \\
level $\mathbf{n = 6 9}$ & $\mathbf{N}(\boldsymbol{\%})$ & $\mathbf{E c l a m p s i a}$ & $\mathbf{A R F}$ & IUGR & IUFD & Birth asphyxia & Severe HTN \\
Present & $3(4.34)$ & $13(18.8)$ & $5(7.24)$ & $9(13)$ & $6(8.7)$ & $15(21.8)$ & $61(88.4)$ \\
\hline Absent & $66(95.7)$ & $56(81.2)$ & $64(92.76)$ & $60(87)$ & $63(91.3)$ & $54(78.2)$ & $8(11.6)$ \\
\hline P value & $\mathrm{P}=0.46$ & $\mathrm{P}=0.03 *$ & $\mathrm{P}=0.27$ & $\mathrm{P}=0.13$ & $\mathrm{P}=0.07$ & $\mathrm{P}=0.001^{*}$ & $\mathrm{P}=0.03 *$ \\
\hline
\end{tabular}

Fisher's exact test * signifies statistically significant $(\mathrm{p}<0.05)$

Table 3: Prognostic accuracy scoring for serum uric acid as a biochemical marker in maternal/foetal outcomes in preeclampsia participants.

\begin{tabular}{|llllll|}
\hline Pregnancy outcome/ serum marker & Sensitivity & Specificity & PPV & NPV & Accuracy \\
\hline Severe Hypertension & 85 & 50 & 87.1 & 45.45 & 0.78 \\
\hline HELLP & 100 & 22.68 & 3.84 & 100 & 0.25 \\
\hline Eclampsia & 100 & 25.29 & 16.67 & 100 & 0.13 \\
\hline Acute renal failure & 100 & 23.15 & 6.41 & 100 & 0.27 \\
\hline IUGR & 90.91 & 23.59 & 12.82 & 95.45 & 0.31 \\
\hline IUFD & 85.71 & 22.58 & 22.22 & 95.65 & 0.27 \\
\hline Birth Asphyxia & 80.95 & 24 & 22.97 & 81.81 & 0.35 \\
\hline
\end{tabular}

Relationship between elevated uric acid levels and maternal/foetal complications in the preeclampsia patients.

No client in the control group had a high serum uric acid level while $69 \%$ of preeclampsia group had high uric acid levels. Amongst these, 3 (4.34\%) developed HELLP syndrome, 13 (18.84\%) developed eclampsia,5 (7.24\%) developed acute renal failure,61(88.4\%) had severe hypertension, (13\%) of them had babies with IUGR, 6 $(8.69 \%)$ suffered IUFD and 15 (21.75) had babies who suffered birth asphyxia. The association between elevated uric acid and eclampsia, severe hypertension and birth asphyxia were statistically significant with $\mathrm{p}$ values of $0.03,0.03$ and 0.01 respectively. While the association between elevated urea levels and HELLP syndrome, acute renal failure, IUGR and IUFD were not statistically significant $(\mathrm{p}>0.05)$ see (Table 2$)$.

Prognostic accuracy scoring for serum uric acid as a biochemical marker in maternal/foetal outcomes in preeclampsia participants.

The ability of the uric acid marker to predict adverse pregnancy outcomes was measured. In terms of the sensitivity, specificity, positive predictive value (PPV), negative predictive value (NPV) and accuracy of the uric acid marker and calculated as shown in (Table 3) and.

The prognostic accuracy was highest for severe hypertension, low for other outcomes and lowest for eclampsia (Figure 1). 


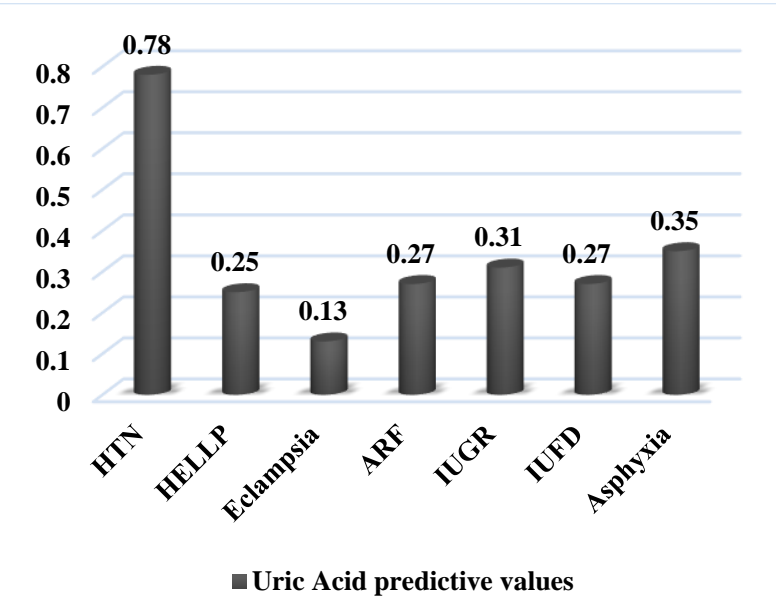

HTN=hypertension, HELLP syndrome, ARF = acute renal failure, IUGR= intrauterine growth retardation, IUFD= intrauterine foetal death, birth asphyxia

Figure 1: Prognostic accuracy of serum uric acid in predicting adverse pregnancy outcomes.

\section{DISCUSSION}

In this study, the mean age of cases and controls were $28 \pm 6.7$ and $31 \pm 6.5$ respectively. The mean age of cases is higher than $27 \pm 4.9$ and $27.2 \pm 5$.6 years reported in calabar and ogun states respectively. ${ }^{17,18}$ The results from this study are consistent with the usual risk factors for preeclampsia including primipaternity and family history of preeclampsia. ${ }^{4}$ Primipaternity was more common in the study group $(70 \%)$ than in the control group (45\%) with the difference being statistically significant $(p=0.00)$. This is in keeping with theories surrounding the origins of preeclampsia identifying pregnancy by a new spouse or partner as a risk factor for the condition. ${ }^{4}$

More women in the study group (35\%) as compared to the control group (7\%) had a family history of hypertensive disease in pregnancy. This is in keeping with genetic factors being implicated in the development of preeclampsia where daughters of women with preeclampsia are about four times more likely to develop the disease than daughter in-laws and it has been established that it is familial. ${ }^{4,19}$

This study reported a significantly higher mean serum uric acid level amongst participants with preeclampsia than in those without preeclampsia. This increased amount of serum uric acid amongst clients with preeclampsia has been reported by several studies and this increased amount is thought to correlate with the severity of the disease condition. ${ }^{5,10,20,21}$ Antenatal patients with renal disease were excluded from taking part in this study and as such, it is unlikely that renal disease would have contributed to the higher levels of serum uric acid amongst the study population.

In this study, majority (94\%) of the patients recruited into the study group were at least 34 weeks gestational age and there was a statistically significant association between high serum uric acid levels and eclampsia, IUGR as well as birth asphyxia $(p<0.05)$. This is in keeping with another study where irrespective of the preeclamptic status of the patient, the serum uric acid levels are bound to rise as pregnancy approaches term. ${ }^{5}$

The severity of preeclampsia was determined by clinical features and/or abnormal laboratory parameters. The adverse pregnancy outcomes in this study were considered as features of severe preeclampsia. The ability of the uric acid marker to predict an adverse pregnancy outcome was measured. The prognostic accuracy of uric acid in predicting pregnancy outcomes was low in this study except for severe hypertension. This corroborates with previous studies where it was reported that although uric acid was significantly elevated in women with preeclampsia compared to the normotensives, it was not a good prognostic indicator of severity and maternal or foetal outcome. ${ }^{12}$

There have been conflicting reports on the ability of serum uric acid to predict adverse pregnancy outcomes. ${ }^{22-}$ 24 The wide variation in gestational ages may have accounted for the different opinions found in these studies. Similar findings concluded that uric acid was not a consistent predictive factor for the development of preeclampsia, but its levels generally increase once the disease manifests and the plasma levels approximately correlates with disease severity. ${ }^{21,23}$ From these findings, we suggest that a combination with other biochemical markers will improve the predictability of pregnancy outcomes in preeclampsia.

\section{Limitation}

This was a hospital-based study. The results may not reflect the findings in other tertiary institutions in Nigeria or the west African sub-region.

\section{CONCLUSION}

The mean serum uric acid was higher amongst participants with preeclampsia than in those without preeclampsia. Serum uric acid was found to be useful prognostic indicator for foetomaternal outcomes in women with preeclampsia. However, the prognostic accuracy of serum uric acid level in predicting maternal and foetal outcomes was low. Therefore, an additional biochemical marker may be useful to improve its prognostic accuracy in pregnancy outcomes of these patients.

\section{ACKNOWLEDGMENTS}

The authors appreciate the patients that participated in this research, and the consultants/residents at the departments of obstetrics \& gynaecology and pathology for their roles in making this research possible. 
Funding: No funding sources

Conflict of interest: None declared

Ethical approval: The study was approved by the Institutional Ethics Committee

\section{REFERENCES}

1. Ekine AA, Jeremiah I, Harry TC, West OL. Factors influencing the prevalence of preeclampsia eclampsia in booked and unbooked patients in NDUTH. World J Med Sci. 2015;3(1):1-14.

2. Adeosun OG, Charles-Davies MA, Ogundahunsi OA, Ogunlewe J. Maternal and neonatal outcomes of preeclampsia in African black women, South West Nigeria. Greener J Med Sci. 2015;5(4):67-76.

3. Kwawukume EY, Emuveyan EE. Comprehensive Obstetrics in the Tropics. Ashante and Hittscher, Damsona. 2002:321-9.

4. Agboola A. Pregnancy induced hypertension, preeclampsia and chronic hypertension. In: Agboola A (ed). Textbook of Obstetrics and Gynaecology for Medical Students, 2nd ed. Heinemann Educational Books plc, Ibadan, Nigeria. 2006;348-59.

5. Sazina M, Khalid UK, Nayyar P. Correlation of serum uric acid with maternal age, parity, and severity of blood pressure in preeclamptic pregnancies. Cont J Med Res. 2008;2:28-34.

6. Rajalaxmi K, Nayak S, Manjulu S. Serum Uric Acid Level in Preeclampsia and its Correlation to Maternal and Fetal Outcome. Int $\mathrm{J}$ Bio Res. 2014;5(1):22-4.

7. Richard JJ, Miguel AL, Eric AG. Uric Acid: A Danger Signal From the RNA World That May Have a Role in the Epidemic of Obesity, Metabolic Syndrome, and Cardiorenal Disease: Evolutionary Considerations. Elsevier Inc J Sem Nephro. 2011;31(5):394-9.

8. Razia S, Selina A, Nasima S. Association of Serum Uric Acid with Preeclampsia: A Case Control Study. Delta Med Col J. 2013;1(2):46-50.

9. Slemons JM, Bogert LJ. The uric acid content of maternal and fetal blood. J Biol Chem. 1917;32:63-9.

10. Azza AM. Level of serum uric acid in patients with preeclampsia compared to controls in Khartoum Teaching Hospital. University of Khartoum Graduate College Medical and Health Studies Board, 2010.

11. Felix E, Olivier I, Pascal F. Blood uric acid level as a marker of increased risk of Eclampsia in Severe Preeclamptic patients: A Cross-Sectional Study in Two Tertiary Hospitals of Yaounde, Cameroon. Health Sci Dis. 2016;17(2):7-11.
12. Joel RL, Beth P, Mark B, James MR. Uric acid as a predictor of adverse maternal and perinatal outcomes in women hospitalized with preeclampsia. J Obstet Gynaecol Can. 2014;36(10):870-7.

13. Kanagasibai S. Biochemical Markers in the prediction of preeclampsia. Are we there yet? Inter J Gynaecol Obstet. 2010;1(12):102-5.

14. Lwamga SK, Lemeshow J. Sample size determination in health studies; a practical manual. Geneva. WHO 1991;15-21.

15. James GP, Bee DE, Fuller JB. Proteinuria; accuracy and precision of laboratory diagnosis by Dipstick analysis. Clin Chem. 1978;24:1934-9.

16. Prince CP, James DR. Analytical Review in Clinical Biochemistry: The measurement of Urate. Ann Clin Biochem. 1988;25:484-98.

17. Kooffrey ME, Ekoh M, Ekpoudom DO. The prevalence of preeclampsia among pregnant women in University of Calabar Teaching Hospital, Calabar. Saudi J Health Sci. 2014;3(3):133-6.

18. Sotunsa J, Sharma S, Imaralu J, Lee T, Adepoju A. The hypertensive disorders of pregnancy in Ogun State, Nigeria: Preeclampsia in low and middle income countries. Pregnancy Hypertension: Int J Women's Cardio Health. 2016;6(3):209

19. Yakassai IA, Morhason-Bello IO. Risk factors for preeclampsia among women at antenatal booking in Kano, Northern Nigeria, Nigeria. J Heal Car low Resour Settin. 2013;1(1):46-9.

20. Asgharnia M, Mirblouk F, Kazemi S, Pourmarzi D. Maternal serum uric acid level and maternal and neonatal complications isn preeclamptic women: A cross-sectional study. Int J Reprod Biomed. 2017;15(9):583-8.

21. Prathap T, Kondareddy T. Uric acid as an important biomarker in hypertensive disorders in pregnancy. Int J Reprod Contracept Obstet Gynaecol. 2016;5(12):4382-4.

22. AjZquez-RodrAquez JG, Rico-Trejo EI. Role of Uric Acid in Preeclampsia-Eclampsia. Gynecol Obstet Mex. 2011;79(5):292-7.

23. Paternoster DM, Stella A, Plebani M, Gambaro G, Grella PV. Predictive markers of pre-eclampsia in hypertensive disorders of pregnancy. Int $\mathbf{J}$ Gynecol Obstet. 1999;66(3):237-43.

24. Martin AC, Brown MA. Could Uric Acid have a pathogenic Role in Preeclampsia? Nat Rev Nephro. 2010;6(12):744-8.

Cite this article as: Obagah L, Kasia BE, Jeremiah I, Allagoa DO, Aigere EOS, Kotingo EL, et al. Serum uric acid: a biochemical prognostic indicator of pregnancy outcomes among pre-eclampsia patients at the federal medical centre, Yenagoa. Int J Reprod Contracept Obstet Gynecol 2020;9:4344-9. 